\title{
Metabolic changes induced by TGF- $\beta 1$ via reduced expression of phosphatidylserine decarboxylase during myofibroblast transition
}

\author{
Nobuhiko Uchida, ${ }^{1}$ Yasuo Shimizu, ${ }^{1, *}$ Mio Fujimaki, ${ }^{1}$ Yasuhiro Horibata, ${ }^{2}$ Yusuke Nakamura, ${ }^{1}$ Yukiko Horigane, ${ }^{1}$ \\ Kazuyuki Chibana, ${ }^{1}$ Akihiro Takemasa, ${ }^{1}$ Hiroyuki Sugimoto, ${ }^{2}$ and Seiji Niho ${ }^{1}$
}

${ }^{1}$ Department of Pulmonary Medicine and Clinical Immunology and ${ }^{2}$ Department of Biochemistry, Dokkyo Medical University School of Medicine, 880 Kitakobayashi, Mibu, Tochigi 321-0293, Japan

(Received 1 September, 2021; Accepted 5 October, 2021)

\begin{abstract}
Metabolic alteration is increasingly recognized as an important pathogenic process that underlies fibrosis across many organ types, and metabolically targeted therapies could become important strategies for reducing fibrosis. In present study, target enzymes that are involved in changes in phospholipid metabolism during fibroblast-to-myofibroblast transition induced by transforming growth factor beta 1 (TGF- $\beta 1$ ) were examined. Different amounts of phospholipids were found in the 2 groups. In response to TGF- $\beta 1$ stimulation, 17 lipids decreased and 17 increased. The latter included the phospholipids phosphatidylcholine (PC), phosphatidylserine (PS), and phosphatidylethanolamine (PE). Furthermore, among the rate-limiting enzymes that regulate these phospholipids, phosphatidylserine decarboxylase (PISD) - which controls conversion of PS to PE and is localized in mitochondria-decreased in response to TGF- $\beta 1$. Knockdown of PISD alone without TGF- $\beta 1$ stimulation increased expression of $\alpha$-smooth muscle actin mRNA and production of total collagen. Taken together, these results indicate that PISD is involved in the mechanism of fibrogenesis by regulating phospholipid metabolism.
\end{abstract}

Key Words: phosphatidylserine decarboxylase, myofibroblasts, fibrosis, lung, mitochondria, phospholipids

F ibrosis of an organ causes it to dysfunction, which can make a disease incurable. ${ }^{(1)}$ The underlying causes of organ fibrosis have been reported as chronic inflammation, senescence, and genetics in organs such as lung, liver, kidney, heart, and pancreas. ${ }^{(1-3)}$ Many studies have identified transforming growth factor beta 1 (TGF- $\beta 1$ ) as a critical signaling cascade in organ fibrogenesis, and myofibroblasts (MFB) have been shown to be a key effector in fibrosis. ${ }^{(1,4)}$ In pulmonary fibrosis, MFB are involved in various processes, such as secretion, migration, accumulation, and differentiation of collagen to form a scar, ${ }^{(5-7)}$ and MFB that express $\alpha$-smooth muscle actin ( $\alpha$ SMA) contribute to increased production of the extracellular matrix. ${ }^{(8)}$ Pulmonary fibrosis is a highly common and fatal pathological response in pulmonary diseases, and mortality rates are high for both idiopathic pulmonary fibrosis and progressive fibrosing interstitial lung disease. ${ }^{(9)}$ Recently, pulmonary fibrosis has been playing a critical role as one of the sequelae of severe acute respiratory syndrome coronavirus 2 infection. ${ }^{(10)}$ In bronchial asthma, MFB also play crucial roles in bronchial wall remodeling, a process that makes the disease more difficult to control. ${ }^{(11)}$

Only a few anti-fibrotic agents are currently available to treat pulmonary fibrosis, making treatment for fibrosis an urgent unmet need..$^{(5,12)}$ Metabolic alteration has been increasingly recognized as an important pathogenic process that underlies fibrosis across many organ types, and metabolically targeted therapies could become important strategies for reducing fibrosis. ${ }^{(13)}$ TGF- $\beta 1$ is a potent metabolic regulator that affects mitochondrial biogenesis and biogenetics during the transition of fibroblasts (FB) to MFB. ${ }^{(14)}$ Mitochondrial dysfunction and metabolic reprogramming have been reported as drivers of pulmonary fibrosis via glycolysis or fatty acid oxidation. ${ }^{(15,16)}$ Mitochondrial function requires preservation of normal lipid composition, which in turn depends on the capacity of mitochondria to transport phospholipids from the endoplasmic reticulum (ER) or to synthesize phospholipids, ${ }^{(17)}$ i.e., the metabolic pathways of phosphatidylcholine (PC) to phosphatidylserine (PS) and/or phosphatidylethanolamine (PE) to PS in the ER and PS to $\mathrm{PE}$ in the mitochondria. ${ }^{(18)}$ The phospholipid composition of cells changes as cell morphology changes in the processes of cell differentiation, invasion, and network formation. ${ }^{(19-21)}$ Changes in lipid metabolism related to mitochondria have been demonstrated in human lung tissue from patients with idiopathic pulmonary fibrosis or murine alveolar type II cells. ${ }^{(22,23)}$ However, to our knowledge no studies have examined the role of phospholipids in FB-to-MFB transition. Therefore, this study aimed to identify the phospholipids that are involved in FB-to-MFB transition and to investigate the underlying mechanisms of metabolic changes with the aim to identify a novel target for treatment of lung fibrosis.

\section{Materials and Methods}

First, we performed phospholipid lipidomics analysis on TGF$\beta 1$-induced FB-to-MFB transition of human lung fibroblasts. On the basis of the results of this analysis, we studied the expression of enzymes related to the target metabolic pathway. Last, we examined the potential of TGF- $\beta 1$ to induce fibrogenesis. Details are provided below.

Cell culture. A flow diagram of the experimental procedure is shown in Fig. 1. Human fetal lung fibroblasts (HFL1, American Type Culture Collection, ATCC) were cultured on collagen-coated tissue culture plates (IWAKI, Tokyo, Japan) in Ham's F-12K medium (Thermo Fisher Scientific, Cleveland, $\mathrm{OH})$ with $10 \%$ fetal bovine serum and $1 \%$ penicillin/streptomycin (Thermo Fisher Scientific) at $37^{\circ} \mathrm{C}$ in a humidified $5 \%$ $\mathrm{CO}_{2}$ atmosphere, as previously described. ${ }^{(20)}$ When the cells had grown to subconfluent conditions ( $\sim 80 \%$ confluent), they were washed 3 times with phosphate-buffered saline (PBS) at $37^{\circ} \mathrm{C}$.

*To whom correspondence should be addressed.

E-mail: yasuo-s@dokkyomed.ac.jp 


\begin{tabular}{l}
$\begin{array}{l}\text { TGF- } \beta 1 \text { stimulation of HFL1 } \\
\text { (FB-to-MFB transition) }\end{array}$ \\
Phospholipid lipidomics analysis \\
and extraction of valuable lipids \\
\hline Enzymes related to lipid synthesis pathways \\
\hline Target enzyme \\
$\quad$ Evaluation of enzyme potency for contributing to fibrosis \\
\hline
\end{tabular}

Fig. 1. Flow diagram of the experimental procedure.

The culture medium was then replaced with serum-free medium and maintained for $24 \mathrm{~h}$ before stimulation with recombinant human TGF- $\beta 1$ (R\&D systems, Minneapolis, MN). To confirm that the conditions were suitable for FB-to-MFB transition, HFL1 were stimulated with 0,1 , and $10 \mathrm{ng} / \mathrm{ml} \mathrm{TGF}-\beta 1$ and cells were harvested at $0,24,48$, and $72 \mathrm{~h}$, as previously described. ${ }^{(24)}$ The maximum expression of $\alpha \mathrm{SMA}$ was found at the $10 \mathrm{ng} / \mathrm{ml}$ concentration of TGF- $\beta 1$ at $48 \mathrm{~h}$ (Supplemental Fig. $1 *$ ). Therefore, for the experiments described below HFL1 were stimulated with $10 \mathrm{ng} / \mathrm{ml}$ of TGF- $\beta 1$ and cells were harvested at $48 \mathrm{~h}$. To inhibit TGF- $\beta 1$ stimulation, anti-TGF- $\beta 1$ neutralization antibody (R\&D systems) was used at a concentration of $300 \mathrm{ng} / \mathrm{ml}$ according to the manufacturer's instructions. The morphology of HFL1 was assessed by microscopy (BZ-X; KEYENCE, Osaka, Japan), as previously described. ${ }^{(20)}$

Purification of lipids and liquid chromatography-mass spectrometry analysis. Total lipids were extracted from cells, and samples were treated and stored, as previously described.(20) Before lipid extraction, the amount of protein in samples was measured by BCA Protein Assay $\mathrm{Kit}^{\circledR}$ (Thermo Fischer Scientific). Before lipids were analyzed by liquid chromatographytandem mass spectrometry (LC-MS/MS), the amount of lipid was adjusted according to the measured protein assay in the harvested cells. Briefly, cells were grown on a $60-\mathrm{mm}$ dish until subconfluent, washed twice with normal saline, harvested with a cell scraper on ice, and then sonicated. The protein solution (100 $\mu \mathrm{g})$ was mixed with methanol and chloroform in a glass tube (the ratio of solution to methanol to chloroform was $900 \mu \mathrm{l}: 2 \mathrm{ml}: 1 \mathrm{ml})$. After separation in a vortex and centrifuge, lipids were collected, and the samples were stored at $-80^{\circ} \mathrm{C}$ after sealing under nitrogen. Lipids were analyzed by reverse-phase HPLC using an L-column 2 ODS column $(3 \mu \mathrm{m}, 2.0 \times 150 \mathrm{~mm})$ (Chemicals Evaluation and Research Institute, Tokyo, Japan) coupled to a 5500 QTRAP mass spectrometer (Sciex Inc., Foster City, CA). PC and SM were detected in multiple reaction monitoring mode by selecting the $\mathrm{m} / \mathrm{z}$ of the lipid molecular species at Q1 and the precursor ion of $m / z 184$ at Q3 in positive ion mode. PE and pPE were detected by monitoring a precursor ion $m / z 196$ at Q3 in negative ion mode. For detection of PS, neutral loss of $\mathrm{m} / \mathrm{z} 87$ in negative mode was monitored. Data processing was performed with a software (Analyst ${ }^{\mathbb{B}} 1.7$ with HotFix3, Sciex Inc.).

Identification of lipids affected by TGF- $\beta 1$ stimulation of HFL1. To ascertain the expression levels of detected phospholipids, we extracted phospholipids showing a peak height greater than 1,000 intensity and a peak area greater than 1,000 intensity. The largest total variance of the samples was assessed by principal component analysis-discriminant analysis (PCA-DA), which maximizes the separation between sample groups while minimizing the variation within groups. PCA-DA score was performed on sample groups from 0 -h and 48 -h cell cultures with $[$ TGF- $\beta 1(+)]$ and without [TGF- $\beta 1(-)]$ TGF- $\beta 1$ stimulation. Furthermore, a PCA-DA blot was performed for all samples together, i.e., without accounting for the culture time $(0$ or $48 \mathrm{~h})$ or stimulation condition TGF- $\beta 1(+)$ or TGF- $\beta 1(-)$. PCA-DA blot was able to divide the samples into several groups on the basis of the lipid levels. For lipids showing statistically different levels $(p<0.05)$ between TGF- $\beta 1(+)$ and TGF- $\beta 1(-)$ in PCA-DA score, we compared the change in the ratio of these lipids in the TGF- $\beta 1(+)$ and TGF- $\beta 1(-)$ conditions. If lipids showed a decrease or increase in the TGF- $\beta 1(+)$ to TGF- $\beta 1(-)$ ratio, another PCA-DA blot was performed to confirm whether the two groups could still be separated.

Real-time quantitative polymerase chain reaction. Real time semi-quantitative polymerase chain reaction (RT-PCR) was performed with a Takara PCR Thermal Cycler TP3000 (TaKaRa Bio Inc., Shiga, Japan), as previously described. ${ }^{(20)}$ Briefly, the cells stimulated with TGF- $\beta 1$ were collected, and RNA was extracted by TRIzol reagent (Thermo Fisher Scientific). Before RT-PCR, RNA was quantified by Nanovue Plus (GE Healthcare, Chicago, IL), and a total of $1 \mu \mathrm{g}$ was subjected to RT-PCR. Total RNA was reverse transcribed into cDNA with the PrimeScript ${ }^{\mathrm{TM}}$ RT Reagent Kit (TaKaRa Bio Inc.) with oligo dT and random primers. RT-PCR was performed with each of these cDNAs with the TB Green Premix Ex Taq kit (TaKaRa Bio Inc.). The sequence primers used for amplification were as follows: glyceraldehyde-3-phosphate dehydrogenase (GAPDH), forward TCAACAGCGACACCCACTCCT, reverse TGAGGTCCACCA CCCTGTTG; $\alpha$ SMA, forward CTGTTCCAGCCATCCTTCAT, reverse TCATGATGCTGTTGTAGGTGGT; phosphatidylserine decarboxylase (PISD), forward CTGAGCCAATCCCTA CAGCC, reverse TACAAAGCCACCTCCCAGTG; phosphatidylserine synthase 1 (PSS1), forward TGTTAGCTTTCC CCAATGGA, reverse GCAAAAATATCAAAGTGGCTGAT; phosphatidylserine synthase 2 (PSS2), forward GAGTCCGAG GTCTACGACGA, reverse TGGCCACAATACCTCTCTTGG; and collagen-1, forward GGGATTCCCTGGACCTAAAG, reverse GGAACACCTCGCTCTCCA. The PCR condition was 1 cycle at $95^{\circ} \mathrm{C}$ for $30 \mathrm{~s}, 1$ cycle at $95^{\circ} \mathrm{C}$ for $5 \mathrm{~s}$, and 50 cycles at $60^{\circ} \mathrm{C}$ for $30 \mathrm{~s}$ each. The levels of mRNA expression were given relative to GAPDH.

Immunoblot blot analysis. To determine suitable conditions for evaluating lipid changes in FB-to-MFB transition stimulated by TGF- $\beta 1$, we performed an immunoblot for $\alpha \mathrm{SMA}$ protein. Cells were grown on a $60-\mathrm{mm}$ dish and harvested in $300 \mu \mathrm{l}$ of cell lysis buffer (Cell Signaling Technology, Beverly, MA), and the protein content was measured by BCA Protein Assay Kit (Thermo Fischer Scientific). For immunoblotting, $4 \mu \mathrm{g}$ of the protein was loaded onto each well. The antibodies used in this study were anti- $\alpha$ SMA (Abcam, Cambridge, MA), antiGAPDH (IMGENEX, San Diego, CA), and anti-PISD antibody [molecular weight $(\mathrm{MW})=46 \mathrm{kDa}$, observed PISD $\mathrm{MW}=50$ $55 \mathrm{kDa}$, according to the manufacturer; Proteintech, Rosemont, IL]. The primary antibodies were diluted according to the manufacturers' recommendations. As a secondary antibody, anti-rabbit alkaline-phosphatase conjugated antibody (secondary antibody solution Alk-Phos.conjugated; Thermo Fisher Scientific) was used. Blocking was performed with commercially available blocking solution (WesternBreeze ${ }^{\mathrm{TM}}$ Blocker/Diluent, Part A and B; Thermo Fisher Scientific). The immunoblot procedures were performed as previously described, ${ }^{(25)}$ and the signals were detected with an immunoblot system (BIO-RAD ChemiDoc XRS-J/Quantity One ver. 4.6 or BIO-RAD ChemiDoc Touch 
Imaging System/Image Lab Touch Software ver. 2.2.0.08; BioRad Laboratories, Hercules, CA).

Small interfering-RNA for PISD. To assess the effect of knockdown of PISD on FB-to-MFB transition, RNA interference on PISD was performed in HFL1. Three small interfering RNAs (si-RNAs) that target different sites on PISD were purchased (Invitrogen, Carlsbad, CA), and the one that showed the most potent knockdown of PISD was used. The specific sense and antisense si-RNAs used in the study were as follows: sense, GAAGAACUGUGAGGUGGAGCAGGUA; antisense, UACCUGCUCCACACAGUUCUUC. As a control, Stealth RNAi Negative Control Duplex (Invitrogen) was used. Cultured HFL1 were resuspended with F-12K medium without anti-biotic and anti-mycotic agents and spread on 6-well plate dish (Falcon plates; Corning Inc., Corning, NY) at a concentration of $1.5 \times$ $10^{5} / \mathrm{ml}$ in each well. After $24 \mathrm{~h}$, si-RNAs were transfected into cells by using Lipofectamine RNAiMAX (Thermo Fisher Scientific), according to the manufacturer's instructions. After $48 \mathrm{~h}$, the cells were harvested for RT-PCR and immunoblotting for PISD and $\alpha$ SMA.

Total collagen assay. To ascertain the contribution of PISD knockdown to functional fibrogenesis, collagen-1 mRNA production was examined by treating HFL1 with si-PISD. After $48 \mathrm{~h}$, cell pellets were collected and the culture medium was treated by acid pepsin extraction for $24 \mathrm{~h}$. Ass a positive control, TGF- $\beta 1$-treated samples were also compared. Extracted total collagen, i.e., type I to IV, was measured by colorimetric analysis, according to the manufacturer's instructions (Siricol Soluble Collagen Assay Kit; Biocolor Life Science Assays, Carrickfergus, UK).

Statistical analysis. Mean values and SEM were calculated. Student's $t$ test was used to compare two groups, and one-way analysis of variance followed by Bonferroni analysis was used to compare three or more groups. Analyses were performed with Statview software (ver. 5.0; Abcas Concepts, Inc., Berkley, CA). LC-MS/MS data were analyzed as described above. Statistical significance was set at $p<0.05$.

\section{Results}

Expression of $\alpha$ SMA protein in HFL1 stimulated by TGF- $\beta 1$ and morphological changes in FB-to-MFB transition. The expression of $\alpha$ SMA (42 kDa) was highest at $48 \mathrm{~h}$ with $10 \mathrm{ng} / \mathrm{ml}$ of TGF- $\beta 1$ (Fig. 2A). During the experiments, levels of GAPDH $(36 \mathrm{kDa})$ did not change over time (Fig. 2B). To confirm the contribution of TGF- $\beta 1$ to expression of $\alpha$ SMA, anti-TGF- $\beta 1$ antibody was added to the cell culture. After $48 \mathrm{~h}$, expression of $\alpha$ SMA protein was reduced by $300 \mathrm{ng} / \mathrm{ml}$ anti-TGF- $\beta 1$ antibody (Fig. 2C). In these experiments, the expression of GAPDH was not affected (Fig. 2D). To further confirm the results of immunoblot analysis, samples were examined for morphological changes by microscopic analysis. Compared with the cell culture at $0 \mathrm{~h}$ (Fig. 2E) and $48 \mathrm{~h}$ without TGF- $\beta 1$ stimulation (Fig. $2 \mathrm{~F}$ ), TGF- $\beta 1$ stimulation $(10 \mathrm{ng} / \mathrm{ml})$ induced morphological changes in the shape of FB, i.e., they changed from spindle to stellate, and markedly reduced cell-cell contact (Fig. 2G). Neutralization with anti-TGF- $\beta$ antibody partially blocked these morphological changes (Fig. 2H).

Phospholipid lipidomics analysis of TGF- $\beta 1$-stimulated FBto-MFB transition. Because of the results of the experiments on $\alpha$ SMA expression and morphological changes after TGF- $\beta 1$ stimulation of HFL1, lipid extraction was performed at $48 \mathrm{~h}$ after stimulation with $10 \mathrm{ng} / \mathrm{ml}$ of TGF- $\beta 1$. Subsequent LC-MS/MS analysis detected 117 kinds of phospholipids, and PCA-DA score
A
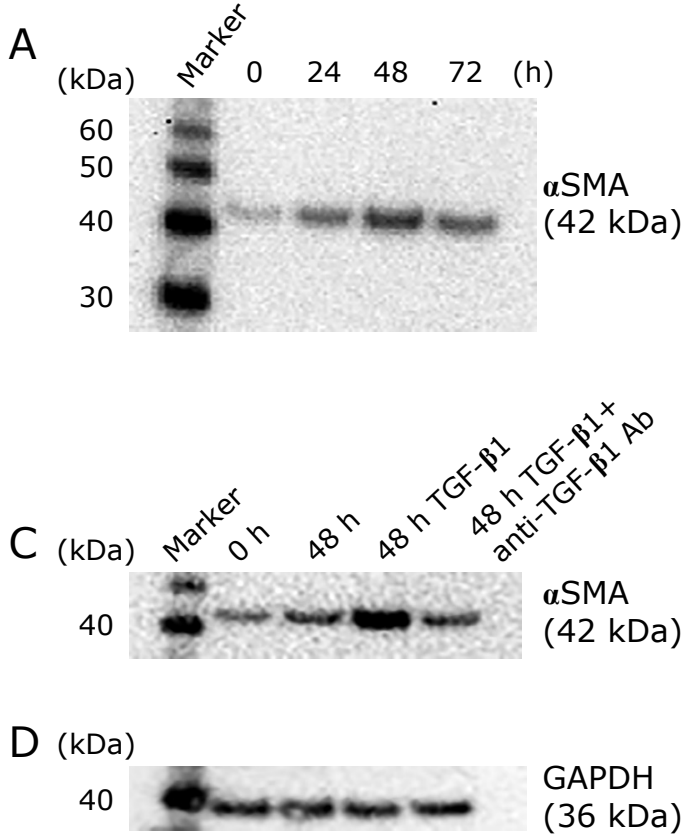

B
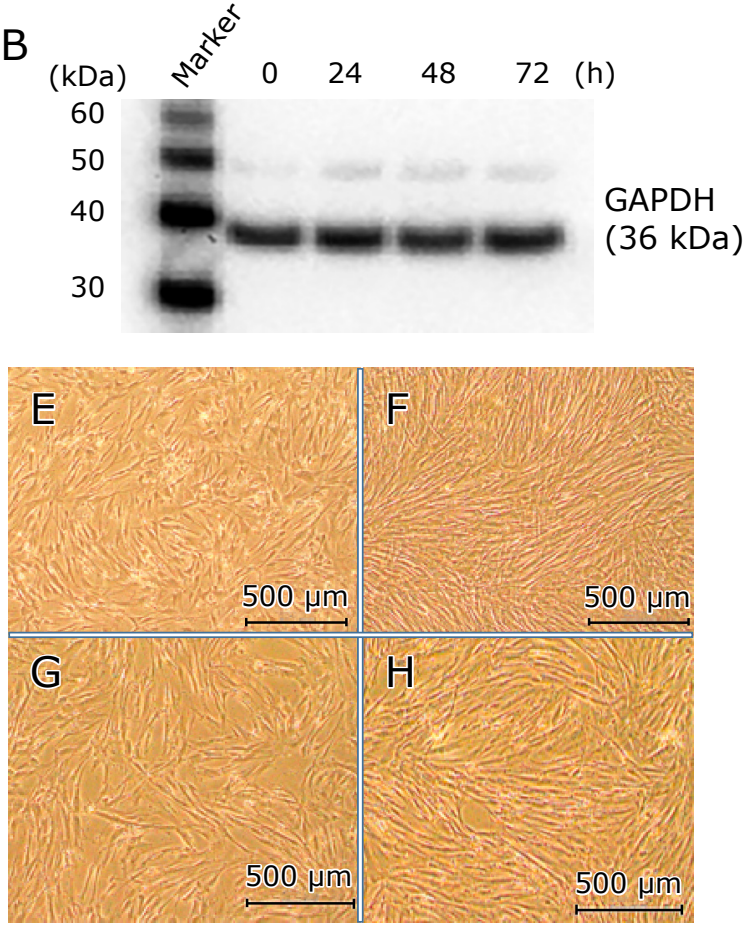

Fig. 2. Time course of $\alpha$-smooth muscle actin protein expression during fibroblast-to-myofibroblast transition of human fetal lung fibroblasts. (A) The maximum expression of $\alpha$-smooth muscle actin ( $\alpha \mathrm{SMA} ; 42 \mathrm{kDa}$ ) was at $48 \mathrm{~h}$ after stimulation with $10 \mathrm{ng} / \mathrm{ml}$ of transforming growth factor beta 1 (TGF- $\beta 1$ ). (B) The expression of glyceraldehyde-3-phosphate dehydrogenase (GAPDH; $36 \mathrm{kDa}$ ) over time during fibroblast (FB)-to-myofibroblast (MFB) transition. (C, D) Expression of $\alpha$ SMA (C) and GAPDH (D) after treatment with TGF- $\beta 1$ or with TGF- $\beta 1$ and anti-TGF $\beta 1$ neutralizing antibody. Lane 1; molecular weight marker, lane 2; $0 \mathrm{~h}$, lane 3; $48 \mathrm{~h}$ after TGF- $\beta 1$ stimulation, and lane $4 ; 48 \mathrm{~h}$ after TGF- $\beta 1$ stimulation and anti-TGF- $\beta 1$ treatment. (E-H) Morphological changes of human fetal lung fibroblasts during TGF- $\beta 1$-induced FB-to-MFB transition. Panel E; 0 h, panel F; $48 \mathrm{~h}$, panel G; after stimulation with TGF- $\beta 1(10 \mathrm{ng} / \mathrm{ml})$, and panel $\mathrm{H}$; after stimulation with TGF- $\beta 1(10 \mathrm{ng} / \mathrm{ml})$ plus treatment with anti-TGF- $\beta 1$ antibody $(300 \mathrm{ng} / \mathrm{ml})$. Scale bar: $500 \mu \mathrm{m}$. 
A
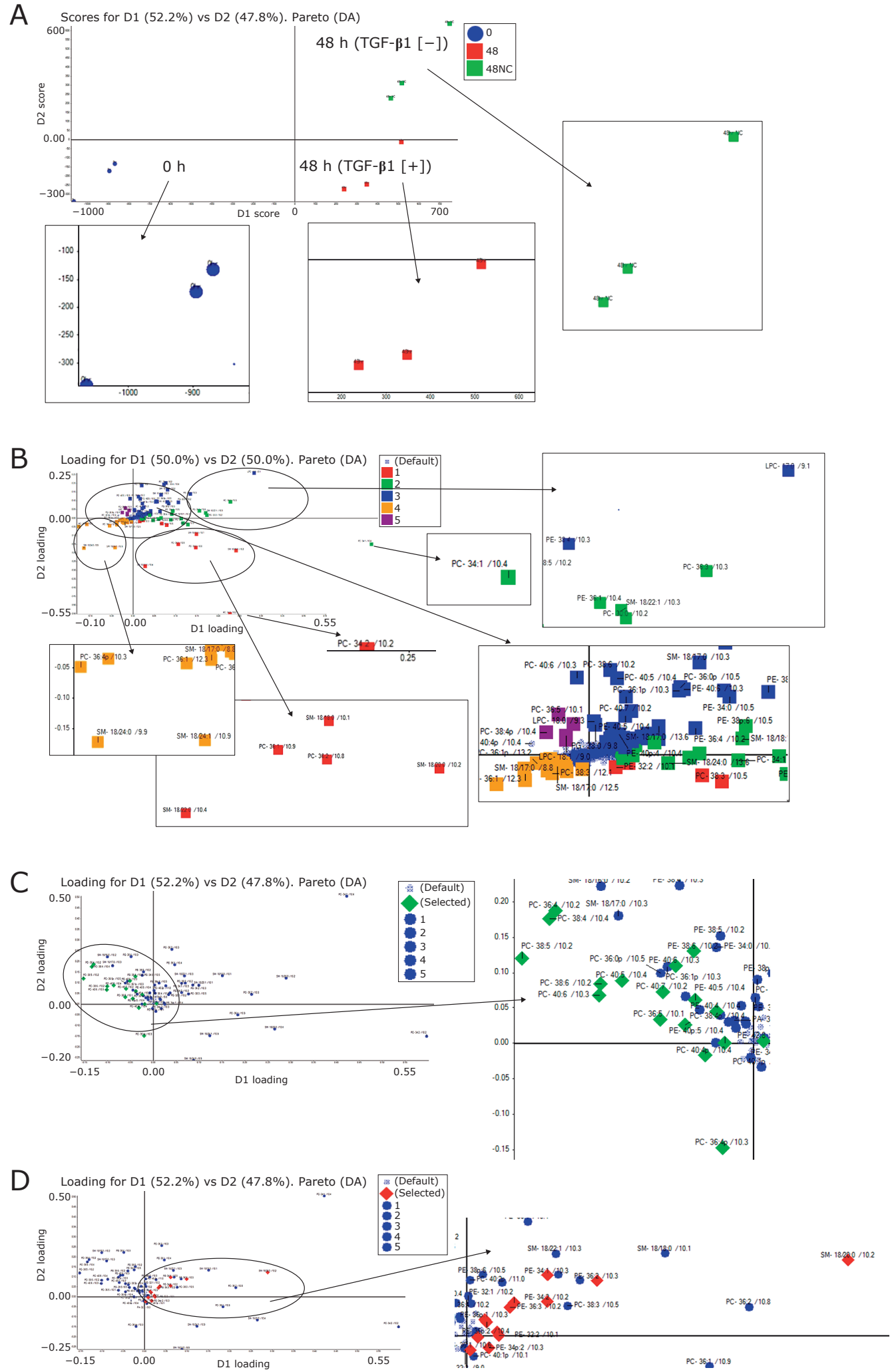

Fig. 3. Principal component analysis-discriminant analysis. (A) Principal component analysis-discriminant analysis (PCA-DA) score of the lipid metabolites in human fetal lung fibroblasts (HFL-1) in the control $(0 \mathrm{~h})$ and $48 \mathrm{~h}$ after transforming growth factor beta 1 (TGF- $\beta 1$ ) stimulation [TGF$\beta 1(+)$ ] or no stimulation [TGF- $\beta 1$ (-)]. (B) PCA-DA blot for entire sample without grouping, i.e., without accounting for time (0 or $48 \mathrm{~h})$ or stimulation (with or without TGF- $\beta 1$ ). Samples were separated into 5 groups. Among the groups, lipids with a decrease in the ratio of TGF- $\beta 1$ (+) to TGF- $\beta 1$ (-) listed in Table 1 (dark green in the Fig. 3C) were still separate from groups 3 and 4 in the PCA-DA blot analysis shown in Fig. 3B (C), and increased levels of these lipids (red) were separate from groups 1 and 2 shown in Fig. 3B of the PCA-DA blot analysis (D). PC; phosphatidylcholine, $\mathrm{PE}$; phosphatidylethanolamine, PS; phosphatidylserine, SM; sphingomyelin. 
Table 1. Changes of phospholipids detected by liquid chromatography-mass spectrometry during transforming growth factor beta 1-induced fibroblast-to-myofibroblast transition of human fetal lung fibroblasts

\begin{tabular}{l|c|l|l|c|c|}
\multicolumn{1}{c}{ Decrease with TGF- $\beta 1$ treatment } & \multicolumn{2}{c}{ Increase with TGF- $\beta 1$ treatment } \\
\hline Lipids & Ratio (TGF+/TGF-) & $p$ value & Lipids & Ratio (TGF+/TGF-) & $p$ value \\
\hline PC-36:4 & 0.88 & 0.043 & PE-32:1 & 2.33 & 0.006 \\
\hline PC-36p:4 & 0.63 & 0.011 & PE-32:2 & 3.85 & 0.006 \\
\hline PC-38:4 & 0.68 & 0.021 & PE-34:1 & 1.51 & 0.032 \\
\hline PC-36:5 & 0.63 & 0.007 & PE-34:2 & 2.44 & 0.002 \\
\hline PC-38p:4 & 0.76 & 0.032 & PE-34p:2 & 1.86 & 0.02 \\
\hline PC-38:5 & 0.59 & 0.003 & PE-34p:2 & 2.59 & 0.006 \\
\hline PC-38:6 & 0.48 & 0.002 & PE-34p:1 & 2.13 & 0.002 \\
\hline PC-40p:4 & 0.65 & 0.014 & PE-36p:1 & 1.46 & 0.018 \\
\hline PC-40:5 & 0.59 & 0.002 & PE-36:2 & 1.52 & 0.033 \\
\hline PC-40:6 & 0.49 & 0.005 & PE-36p:2 & 2.01 & 0.004 \\
\hline PC-40:7 & 0.44 & 0.004 & PE-36:3 & 1.57 & 0.016 \\
\hline PE-38:6 & 0.65 & 0.019 & PS-34:1 & 2.79 & 0.011 \\
\hline PE-40:4 & 0.83 & 0.035 & PS-36:1 & 2.56 & 0.044 \\
\hline PE-40:5 & 0.68 & 0.005 & PS-36:2 & 2.79 & 0.028 \\
\hline PE-40p:5 & 0.75 & 0.01 & PS-38:1 & 2.5 & 0.046 \\
\hline PE-40:6 & 0.66 & 0.006 & PS-38:2 & 2.76 & 0.006 \\
\hline PE-42:8 & 0.54 & 0.0002 & SM-18/20:0 & 1.31 & 0.049 \\
\hline An & & & & & 2 \\
\hline
\end{tabular}

An increase or decrease in the levels of lipids was determined from the ratio at $48 \mathrm{~h}$ of the amounts of phospholipids after stimulation with transforming growth factor beta 1 (TGF- $\beta 1$ ) to the amounts without TGF- $\beta 1$ stimulation (TGF+ to TGF-). PC; phosphatidylcholine, PE; phosphatidylethanolamine, pPE; plasmalogen-PE, PS; phosphatidylserine, SM; sphingomyelin.

showed clear separation into three groups: TGF- $\beta 1(-)$ at 0 and $48 \mathrm{~h}$ and TGF- $\beta 1(+)$ (Fig. 3A). Metabolites from the TGF- $\beta 1(-)$ samples were localized in D1 score high and D2 score high, whereas metabolites from TGF- $\beta 1(+)$ samples were localized in D1 score low and D2 score high. Among the metabolites that showed a statistically significant difference between TGF- $\beta 1(-)$ and TGF- $\beta 1(+), 34$ matched the criteria of a peak height greater than 1,000 intensity and a peak area greater than 1,000 intensity and a change in the ratio of TGF- $\beta 1(+)$ to TGF- $\beta 1(-)$ (Table 1$)$. The metabolites that showed significant changes were the PC, PS, and PE. Some significant differences were found in phosphatidylglycerol (PG) and sphingomyelin (SM) between samples with and without TGF- $\beta 1$, but these lipids were excluded because of low height or peak area. Seventeen lipids decreased after stimulation, i.e., 11 species of PC and 6 species of PE, and 17 increased, i.e., 11 species of PE, 5 of PS, and 1 of SM. Most of the decreased phospholipids were polyunsaturated phospholipids whereas most of the increased lipids were mono- or di-unsaturated phospholipids. PS only increased and PC only decreased. The plasmalogen type of PE (pPE) was also observed in the increased lipids 34p:1, 36p:1, and 36p:2 and in the decreased lipids 40p:5. The PCA-DA blot that was performed on the whole group of obtained lipids without accounting for sample, i.e., whether 0 or $48 \mathrm{~h}$ or TGF- $\beta 1(-)$ or TGF- $\beta 1(+)$, separated the lipids into 5 groups (Fig. $3 \mathrm{~B}$ ). The lipids shown as decreased or increased in Table 1 were again analyzed by PCADA blot, and the blot still showed distinct groups of decreased (Fig. 3C, dark green diamond shape symbol) and increased (Fig. 3D, red diamond shape symbol) lipids according to the ratio of TGF- $\beta 1(+)$ to TGF- $\beta 1(-)$.

Expression levels of enzyme mRNA involved in metabolic changes in phospholipids and decreased in PISD protein expression by TGF- $\beta 1$. The LC-MS/MS results indicated that the metabolic pathways of PC to PS and/or PE to PS in the ER and PS to PE in mitochondria were responsible for the TGF- $\beta 1$ induced metabolic changes in phospholipids (Fig. 4A). ${ }^{(17)}$ Therefore, we examined whether the mRNA levels of the following enzymes were affected by TGF- $\beta 1$ stimulation: PSS1, which catabolizes PC to PS; PSS2, which catabolizes PE to PS; and PISD, which catabolizes PS to PE. After $48 \mathrm{~h}$ of stimulation with $10 \mathrm{ng} / \mathrm{ml} \mathrm{TGF}-\beta 1$, the mRNA levels of PSS1 (Fig. 4B) and PSS2 (Fig. 4C) were unchanged. However, the expression level of PISD was significantly suppressed by TGF- $\beta 1$ stimulation $[48-\mathrm{h}$ TGF- $\beta 1(+)$ vs TGF- $\beta 1(-)]$ and reversed by neutralizing anti-TGF- $\beta 1$ antibody treatment $[48-\mathrm{h}$ TGF- $\beta 1(+)$ vs $48-\mathrm{h}$ TGF- $\beta 1 \quad(+)$ plus anti-TGF- $\beta 1$ antibody] $(n=3, \quad p<0.05)$ (Fig. 4D). PISD protein expression was examined in HFL1 at $48 \mathrm{~h}$ after stimulation with TGF- $\beta 1$. Compared with no stimulation, TGF- $\beta 1$ stimulation decreased PISD protein expression, as observed at 50 to $55 \mathrm{kDa}$ (calculated MW: 375 amino acids, $43 \mathrm{kDa}$; 408 amino acids, $46 \mathrm{kDa}$; Proteintech), as described in the manufacture's data sheet (Fig. 4E). Relative quantification showed that PISD protein expression was significantly reduced by TGF- $\beta 1$ stimulation $(n=3, p<0.05)$ (Fig. $4 \mathrm{~F})$.

Effects of PISD knockdown on aSMA mRNA. In the first experiment, we tested three purchased si-PISD and found that one significantly knocked down PISD mRNA expression at $48 \mathrm{~h}$ compared with untreated or si-control-treated cells $(n=3$, $p<0.05)$ (Fig. 5A). We also confirmed that PISD protein expression was significantly reduced $(n=3, p<0.05)$ (Fig. $5 \mathrm{~B}$ and $\mathrm{C}$ ). Examination of $\alpha \mathrm{SMA} \mathrm{mRNA}$ and protein expression to ascertain emerging MFB features showed that treatment with si-PISD significantly increased $\alpha$ SMA mRNA expression $(n=3$, $p<0.05$ ) (Fig. 5D) but that $\alpha$ SMA protein levels did not increase (Fig. 5E and F).

Effect of PISD knockdown on total collagen production by HFL1. Collagen-1 mRNA expression in HFL1 was significantly increased by treatment with si-PISD $(n=3, p<0.05)$ (Fig. 6A). 


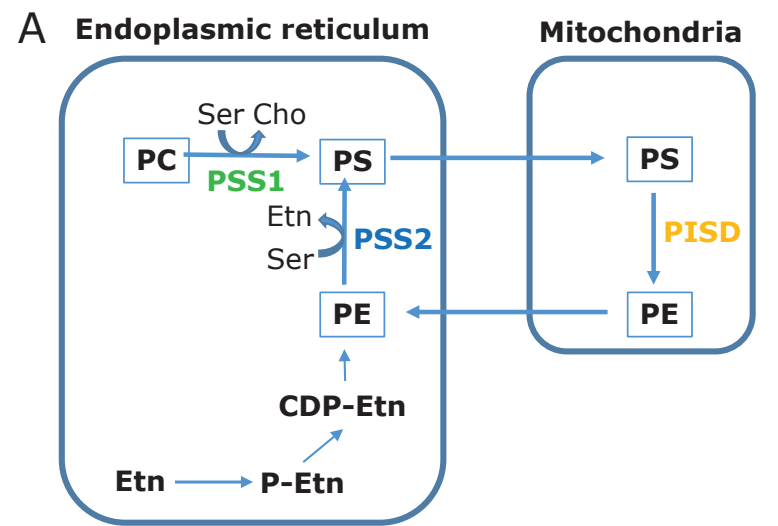

B

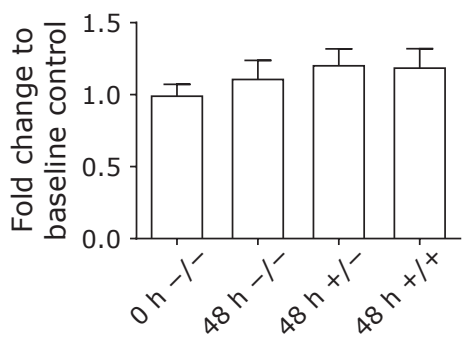

$E$

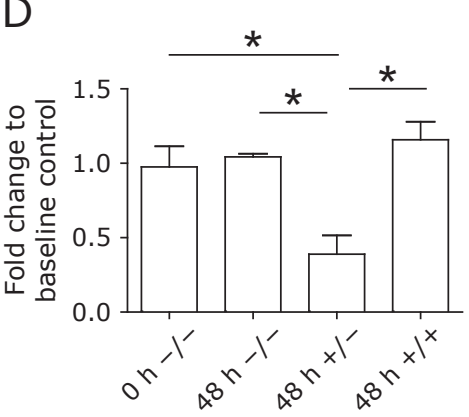

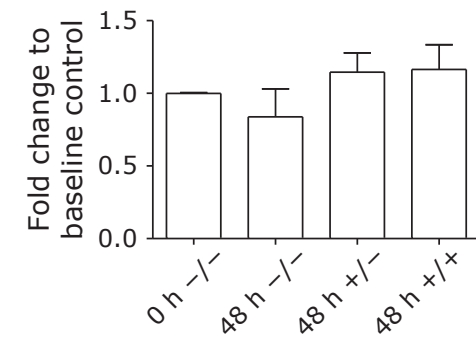

C

$\mathrm{F}$

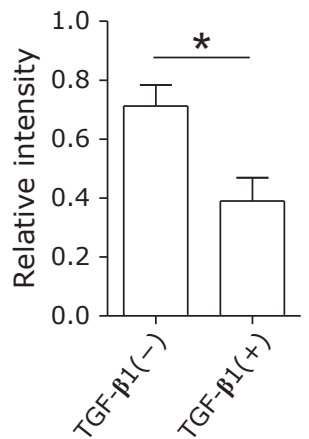

Fig. 4. Metabolic pathways of phospholipids in the endoplasmic reticulum and mitochondria and the expressions of enzymes involved in the pathways. (A) Biosynthesis of phosphatidylethanolamine (PE) takes place in mitochondria by decarboxylation of phosphatidylserine (PS) via phosphatidylserine decarboxylase (PISD). PS can be synthesized from phosphatidylcholine (PC) via phosphatidylserine synthase 1 (PSS1) and from PE via phosphatidylserine synthase 1 (PSS2). (B-D) Expression levels of mRNA for PSS1 (B), PSS2 (C), and PISD (D) (each, $n=3)$. Levels were measured at $0 \mathrm{~h}$ $(0 \mathrm{~h}-/-)$ and $48 \mathrm{~h}(-/-)$ with no stimulation, $48 \mathrm{~h}$ after stimulation with $10 \mathrm{ng} / \mathrm{ml}$ of transforming growth factor beta 1 (TGF- $\beta 1)(48 \mathrm{~h}+/-)$ and after stimulation with $10 \mathrm{ng} / \mathrm{ml}$ TGF- $\beta 1$ plus treatment with $300 \mathrm{ng} / \mathrm{ml}$ anti-TGF- $\beta 1$ antibody $(48 \mathrm{~h}+/+$ ). Levels were indicated as fold change to base line control (0 h -l-). (E) Expression of PISD protein and glyceraldehyde-3-phosphate dehydrogenase (GAPDH) on human fetal lung fibroblasts (HFL-1) at $48 \mathrm{~h}$ with TGF- $\beta 1$ stimulation [TGF- $\beta 1(+)$ ] or without [TGF- $\beta 1$ (-)]. (F) Relative intensity, calculated as PISD/GAPDH, with TGF- $\beta 1$ stimulation [TGF$\beta 1(+)$ ] or without [TGF- $\beta 1(-)](n=3)$. Statistical significance was set at ${ }^{*} p<0.05$. Etn, ethanolamine; CDP-Etn, CDP-ethanolamine; Cho, choline; PISD, phosphatidylserine decarboxylase; PC, phosphatidylcholine; PS, phosphatidylserine; PE, phosphatidylethanolamine; P-Etn, ethanolamine phosphate; PSS, phosphatidylserine synthase; Ser, serine.

In cell pellets, total collagen content was significantly higher after si-PISD treatment compared with untreated and si-control samples, however, it was lower than in the TGF- $\beta 1$-stimulated sample $(n=3, p<0.05)$ (Fig. 6B). In the culture medium, si-PISD increased total collagen production more than in the untreated sample but less than in the TGF- $\beta 1$-stimulated sample $(n=3$, $p<0.05$ ) (Fig. 6C).

\section{Discussion}

In the present study, phospholipid lipidomics analysis of FBto-MFB transition of HFL1 induced by TGF- $\beta 1$ stimulation revealed that levels of the cellular phospholipids PE, PS, and PC increased, as did $\alpha \mathrm{SMA}$ protein expression and total collagen content. On the basis of these results, the expression levels of enzymes related to metabolism of these phospholipids were investigated and the expression level of the enzyme PISD, which catabolizes PS to PE, was found to be reduced. Furthermore, PISD knockdown alone, without TGF- $\beta 1$ stimulation, also increased $\alpha \mathrm{SMA}$ mRNA expression and total collagen content in FB. These results indicated that PISD is involved in the mechanism of FB-to-MFB transition and that reduced PISD expression possibly also plays a role in fibrogenesis.

Among the various pathways for biosynthesis of PE, two are particularly important: the PSD pathway and the cytidine diphosphate (CDP)-ethanolamine pathway. PSD activity is restricted to the mitochondrial inner membrane, with the active site oriented towards the inner membrane space. In the PSD pathway in humans, PE is synthesized by decarboxylation of PS via PISD. Three types of PSD enzymes were identified in different organisms, and PISD was identified in human, mouse and Caenorhabditis elegans. In humans, only one mitochondrial form of PISD was identified. ${ }^{(18,26)}$ In mice, knockout of the PISD gene is embryonically lethal, ${ }^{(27)}$ and in humans, PISD gene variants cause skeletal dysplasia, cataract, white matter changes, the Liberfarb syndrome which is a multisystem disorder affecting eye, ear, bone, and brain development, and spondyloepimetaphyseal dysplasia, in which among other characteristics, the epiphyses are abnormally large. ${ }^{(28,29)}$

The CDP-ethanolamine pathway involves several enzymes, such as ethanolamine kinase (EK), phosphoethanolamine cytidylyltransferase, and 1,2-diacylglycerol choline/ethanolamine phosphotransferase, which mediate synthesis of PE. PE is then catalyzed to PS by PSS1 in the ER, PS can also be synthesized from PC by PSS2, also in the ER. ${ }^{(26)}$ PSS1 and PSS2 have been identified in humans, ${ }^{(30)}$ and mutations in the phosphatidylserine synthase 1 gene encoding PSS1 are causative in Lenz-Majewski syndrome, a rare disease characterized by complex craniofacial, dental, cutaneous, and limb abnormalities combined with intellectual disability. ${ }^{(31)}$ 

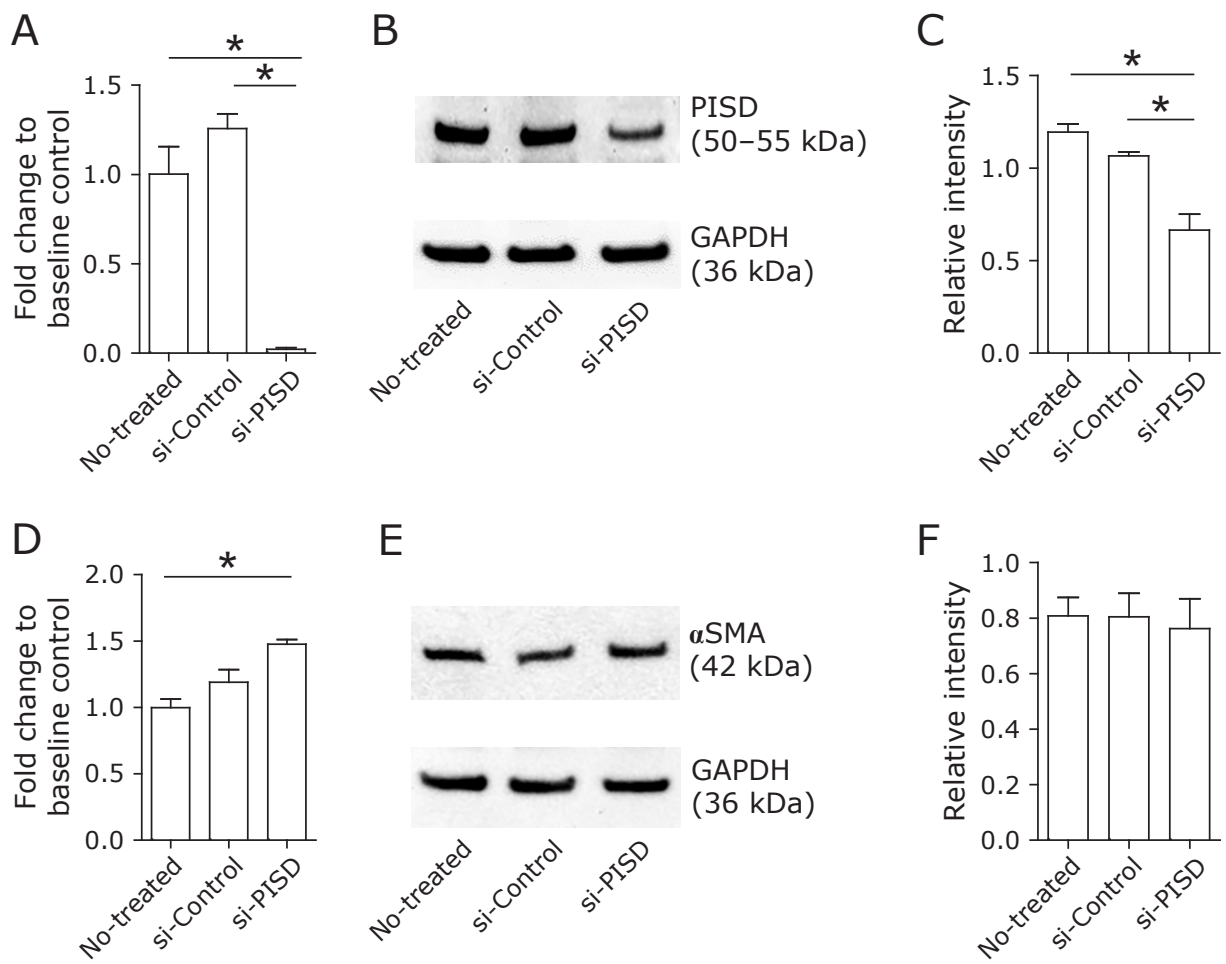

Fig. 5. Effects of phosphatidylserine synthase knockdown on phosphatidylserine synthase and $\alpha$-smooth muscle actin mRNA expressions in human fetal lung fibroblasts. Human fetal lung fibroblasts (HFL1) were treated with small interfering RNA for phosphatidylserine decarboxylase (si-PISD), and the mRNA expression of PISD (A) and $\alpha$-smooth muscle actin ( $\alpha$ SMA) (D) were measured after $48 \mathrm{~h}$ (each, $n=3$ ). The effect of si-PISD on protein expression of PISD (B), aSMA (E), and glyceraldehyde-3-phosphate dehydrogenase (GAPDH) in HFL-1. Levels of mRNA were indicated as fold change to base line control (no-treated). Relative intensity of protein expression was calculated for PISD/GAPDH (C) and $\alpha$ SMA/GAPDH (F) (each, $n=3$ ). Statistical significance was set at ${ }^{*} p<0.05$.

A

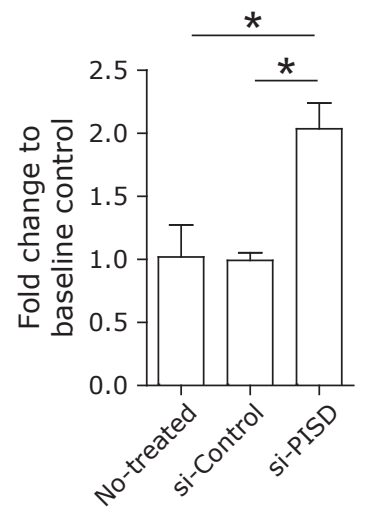

B

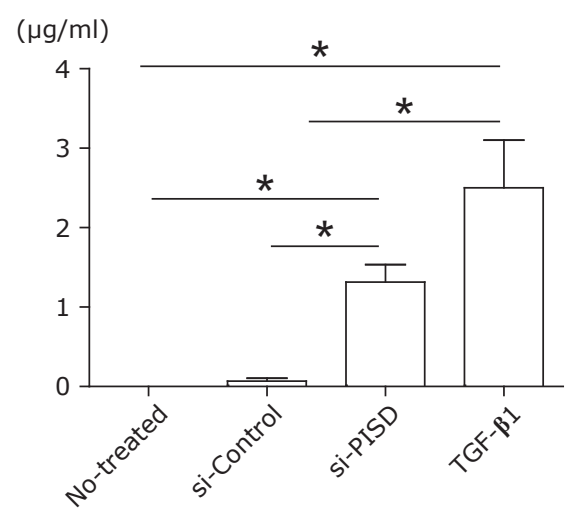

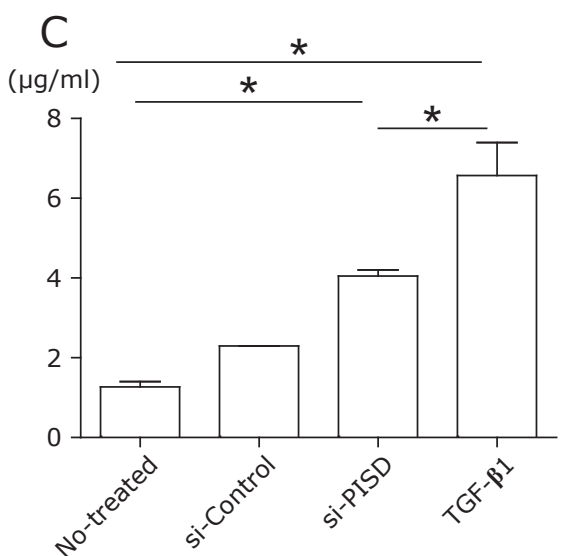

Fig. 6. Phosphatidylserine decarboxylase knockdown effects on collagen-1 mRNA expression in human fetal lung fibroblasts. (A) Effects on human fetal lung fibroblasts (HFL1) at $48 \mathrm{~h}$. The level of collagen-1 mRNA was indicated as fold change to base line control (no-treated). (B, C) Total collagen production after phosphatidylserine decarboxylase (PISD) knockdown of HFL1. Total collagen content in cell pellets (B) and culture medium (C) (each, $n=3$ ) after si-PISD treatment and, as a positive control, transforming growth factor beta 1 (TGF- $\beta 1$ ) stimulation of HFL1 (B). Statistical significance was set at * $p<0.05$.

The present study revealed that among the three enzymes PSS1, PSS2, and PISD. PISD levels decreased in response to TGF- $\beta 1$-induced FB-to-MFB transition of HFL1. A reduced PISD level is consistent with increased PS, indicating decreased biosynthesis of PS to PE in mitochondria. To date, no study has reported that reduced levels of the PISD gene are related to lung fibrosis or that TGF- $\beta 1$-induced metabolic changes in PE, $\mathrm{PS}$, and $\mathrm{PC}$ in the ER are accompanied by increased collagen production. In other organ, similar results have been reported in mouse model of non-alcoholic fatty liver disease. Mitofusin 2 (Mfn2) is mitochondrial membrane protein with a role connecting ER membranes to mitochondria, and binds directly PS. The deficiency of hepatic Mfn2 causes a reduced transfer of PS from ER to mitochondria accompanied with increased PS and reduced PE, and also increased expression of $\alpha \mathrm{SMA}$ and collagen $\alpha-1$ in liver biopsy specimens. In that experiment, 
reduced mRNA expression of PSS1 and PSS2, but not PISD, were significantly lower, suggested that Mfn2 regulated phospholipid biosynthesis through PSS1 and PSS2. ${ }^{(32)}$ The reasons for different results between previous report and present study are unknown. ${ }^{(32)}$ Possible explanations may be different material and methods, such as in vivo mouse liver disease specimen or in vitro human cell line in present study. ${ }^{(32)}$ PISD knockout in mice leads embryonically lethal, however, a study found that $\mathrm{PISD}^{+/-}$ heterozygous mice were born alive and appeared normal, with no reduction in the amounts of PS and PE in tissues. ${ }^{(27)}$ As possible explanations for this finding, the authors discussed compensatory mechanisms, such as PE degradation or increased flux through the CDP-ethanolamine pathway. ${ }^{(27)}$ Although $\mathrm{PISD}^{+/-}$mice appeared to have normal levels of PS and PE, severe lung injury could affect the PISD pathway for PS-to-PE biosynthesis. For example, PE supplementation ameliorated bleomycin-induced lung fibrosis in mice and reduced expression of collagen mRNA and the apoptosis rate of human lung fibroblasts. ${ }^{(33)}$ In the present study, TGF- $\beta 1$-induced FB-to-MFB transition decreased selected polyunsaturated PEs and increased saturated PEs. This finding is in line with a previous report that polyunsaturated PEs were mainly generated by PISD, whereas mono- or di-unsaturated lipids were preferentially generated by the CDP-ethanolamine pathway in mammalian cells. ${ }^{(34)}$ The study suggested that the decrease in polyunsaturated PEs after TGF- $\beta 1$ stimulation was related to reduced PISD expression in mitochondria.

In the present study, in addition to PE, pPE was increased during FB-to-MFB transition of HFL1. Plasmalogens are a unique class of membrane glycerophospholipids containing a fatty alcohol with a vinyl ether bond at the sn-1 position. ${ }^{(35)} \mathrm{pPE}$ is synthesized by peroxisomal matrix enzyme catalysis and structural modification in the ER. ${ }^{(36)}$ Peroxisome is generated from docking of pre-peroxisome vesicles generated by the ER and mitochondria. Unclear is whether PISD regulates the synthesis of pre-peroxisome vesicles. Although the relation between lung fibrosis and pPE has not been reported, levels of pPE in blood mononuclear cells were different in patients with cystic fibrosis than in healthy individuals: Species pPE-38:6 and -40:6 of unsaturated pPE were significantly reduced in the peripheral blood of patients. ${ }^{(37)}$ The present study did not evaluate these unsaturated pPEs or whether the levels of lipid in peripheral blood are reflected in cellular levels. Further investigation is needed about changes in levels of pPEs.

This study has some limitations. First, HFL1 are fetal lung fibroblasts. To elucidate the relation between PISD expression levels and lung fibrosis, we would need to compare PISD expression levels and phospholipid levels in MFB isolated from patients

\section{References}

1 Rockey DC, Bell PD, Hill JA. Fibrosis--a common pathway to organ injury and failure. N Engl J Med 2015; 372: 1138-1149.

2 Garcia CK. Insights from human genetic studies of lung and organ fibrosis. $J$ Clin Invest 2018; 128: 36-44.

3 Jun JI, Lau LF. Resolution of organ fibrosis. J Clin Invest 2018; 128: 97-107.

4 Darby IA, Zakuan N, Billet F, Desmoulière A. The myofibroblast, a key cell in normal and pathological tissue repair. Cell Mol Life Sci 2016; 73: 11451157.

5 Montesi SB, Fisher JH, Martinez FJ, Selman M, Pardo A, Johannson KA. Update in Interstitial Lung Disease 2019. Am J Respir Crit Care Med 2020; 202: $500-507$.

6 Shimizu Y, Dobashi K, Iizuka K., et al. Contribution of small GTPase Rho and its target protein rock in a murine model of lung fibrosis. Am J Respir Crit Care Med 2001; 163: 210-217.

7 Kato K, Logsdon NJ, Shin YJ, et al. Impaired myofibroblast dedifferentiation contributes to nonresolving fibrosis in aging. Am J Respir Cell Mol Biol 2020; 62: 633-644.

8 Barkauskas CE, Noble PW. Cellular mechanisms of tissue fibrosis. 7. New with lung fibrosis and lung fibroblasts from healthy individuals. Second, reduced PC was also observed in TGF- $\beta 1$-induced FBto-MFB transition. PC is synthesized by the CDP-choline pathway via choline phosphotransferase or choline/ethanolamine phosphotransferase, also called the Kennedy pathway, so activation of the Kennedy pathway might have reduced PC in the present study. In another pathway, $\mathrm{PC}$ is synthesized by metabolism of PE via PE $N$-methyltransferase, ${ }^{(38)}$ so when PE $N$ methyltransferase activity is decreased, PC decreases and PE increases; however, this explanation is not in line with the present study. Third, PISD knockdown alone cannot induce increased aSMA protein. Therefore, to elucidate the reason for increased aSMA protein in this study. Forth, the phospholipid accumulation levels and the relation of signal transduction of outer mitochondria, such as kinase pathways linking to $\mathrm{FB}$ to $\mathrm{MFB},{ }^{(1,23)}$ are needed to examine after PISD knockdown. Further studies are needed in the future.

Mitochondrial metabolism may represent a therapeutic target in lung fibrosis. Chronic exposure to environmental triggers, cellular senescence, immunological changes, and virus infection, such as with SARS-CoV-2, are thought to be involved in the mechanisms of mitochondrial dysfunction in the formation of lung fibrosis..$^{(10,15,16,22)}$ Therapeutic approaches for mitochondrial dysfunction, such as mitochondrial transfer, modifying mitochondrial genomes, or chemicals, may have potential in the treatment of these diseases. ${ }^{(39-42)}$

In conclusion, PISD contributes to FB-to-MFB transition of human fetal lung fibroblasts, and this transition is accompanied by changes in phospholipid composition. Regulation of phospholipid metabolism and its responsible enzyme PISD could represent a novel target in pulmonary fibrosis.

\section{Acknowledgments}

The authors thank Prof. D. E. Vance and Prof. J. E. Vance (University of Alberta, Canada) for useful discussions and Kazumi Akimoto (Dokkyo Medical University Research Support Center) for assistance with cell cultures.

\section{Funding}

None.

\section{Conflict of Interest}

No potential conflicts of interest were disclosed. insights into the cellular mechanisms of pulmonary fibrosis. Am J Physiol Cell Physiol 2014; 306: C987-C996.

9 Selman M, Pardo A. When things go wrong: exploring possible mechanisms driving the progressive fibrosis phenotype in interstitial lung diseases. Eur Respir J 2021; 58: 2004507.

10 McDonald LT. Healing after COVID-19: are survivors at risk for pulmonary fibrosis? Am J Physiol Lung Cell Mol Physiol 2021; 320: L257-L265.

11 Michalik M, Wójcik-Pszczoła K, Paw M, et al. Fibroblast-to-myofibroblast transition in bronchial asthma. Cell Mol Life Sci 2018; 75: 3943-3961.

12 Nanthakumar CB, Hatley RJ, Lemma S, Gauldie J, Marshall RP, Macdonald SJ. Dissecting fibrosis: therapeutic insights from the small-molecule toolbox. Nat Rev Drug Discov 2015; 14: 693-720.

13 Zhao X, Kwan JYY, Yip K, Liu PP, Liu FF. Targeting metabolic dysregulation for fibrosis therapy. Nat Rev Drug Discov 2020; 19: 57-75.

14 Bernard K, Logsdon NJ, Ravi S, et al. Metabolic reprogramming is required for myofibroblast contractility and differentiation. J Biol Chem 2015; 290: $25427-25438$

15 Rangarajan S, Bernard K, Thannickal VJ. Mitochondrial dysfunction in 
pulmonary fibrosis. Ann Am Thorac Soc 2017; 14 (Supplement-5): S383S388.

16 Bueno M, Calyeca J, Rojas M, Mora AL. Mitochondria dysfunction and metabolic reprogramming as drivers of idiopathic pulmonary fibrosis. Redox Biol 2020; 33: 101509.

17 Schenkel LC, Bakovic M. Formation and regulation of mitochondrial membranes. Int J Cell Biol 2014; 2014: 709828.

18 Di Bartolomeo F, Wagner A, Daum G. Cell biology, physiology and enzymology of phosphatidylserine decarboxylase. Biochim Biophys Acta Mol Cell Biol Lipids 2017; 1862: 25-38.

19 Shimizu Y, Satou M, Hayashi K, et al. Matrix-assisted laser desorption/ ionization imaging mass spectrometry reveals changes of phospholipid distribution in induced pluripotent stem cell colony differentiation. Anal Bioanal Chem 2017; 409: 1007-1016.

20 Nakamura Y, Shimizu Y, Horibata Y, et al. Changes of plasmalogen phospholipid levels during differentiation of induced pluripotent stem cells 409B2 to endothelial phenotype cells. Sci Rep 2017; 7: 9377.

21 Shimizu Y, Nakamura Y, Horibata Y, et al. Imaging of lysophosphatidylcholine in an induced pluripotent stem cell-derived endothelial cell network. Regen Ther 2020; 14: 299-305.

22 Zhao YD, Yin L, Archer S, et al. Metabolic heterogeneity of idiopathic pulmonary fibrosis: a metabolomic study. BMJ Open Respir Res 2017; 4: e000183.

23 Chung KP, Hsu CL, Fan LC, et al. Mitofusins regulate lipid metabolism to mediate the development of lung fibrosis. Nat Commun 2019; 10: 3390.

24 Utsugi M, Dobashi K, Ishizuka T, et al. C-Jun-NH2-terminal kinase mediates expression of connective tissue growth factor induced by transforming growth factor-beta1 in human lung fibroblasts. Am J Respir Cell Mol Biol 2003; 28: 754-761.

25 Shimizu Y, Dobashi K, Endou K, et al. Decreased interstitial FOXP3 ${ }^{+}$ lymphocytes in usual interstitial pneumonia with discrepancy of CXCL12/ CXCR4 axis. Int J Immunopathol Pharmacol 2010; 23: 449-461.

26 Vance JE. Historical perspective: phosphatidylserine and phosphatidylethanolamine from the 1800 s to the present. J Lipid Res 2018; 59: 923-944.

27 Steenbergen R, Nanowski TS, Beigneux A, Kulinski A, Young SG, Vance JE. Disruption of the phosphatidylserine decarboxylase gene in mice causes embryonic lethality and mitochondrial defects. J Biol Chem 2005; 280: 40032-40040.

28 Girisha KM, von Elsner L, Neethukrishna K, et al. The homozygous variant c.797G $>$ A/p.(Cys266Tyr) in PISD is associated with a Spondyloepimetaphyseal dysplasia with large epiphyses and disturbed mitochondrial function. Hum Mutat 2019; 40: 299-309.

29 Peter VG, Quinodoz M, Pinto-Basto J, et al. The Liberfarb syndrome, a multisystem disorder affecting eye, ear, bone, and brain development, is caused by a founder pathogenic variant in the PISD gene. Genet Med 2019; 21: $2734-2743$.

30 Tomohiro S, Kawaguti A, Kawabe Y, Kitada S, Kuge O. Purification and characterization of human phosphatidylserine synthases 1 and 2. Biochem $J$ 2009; 418: 421-429.

31 Sohn M, Ivanova P, Brown HA, et al. Lenz-Majewski mutations in PTDSS1 affect phosphatidylinositol 4-phosphate metabolism at ER-PM and ER-Golgi junctions. Proc Natl Acad Sci U S A 2016; 113: 4314-4319.

32 Hernández-Alvarez MI, Sebastián D, Vives S, et al. Deficient endoplasmic reticulum-mitochondrial phosphatidylserine transfer causes liver disease. Cell 2019; 177: 881-895.

33 Vazquez-de-Lara LG, Tlatelpa-Romero B, Romero Y, et al. Phosphatidylethanolamine induces an antifibrotic phenotype in normal human lung fibroblasts and ameliorates bleomycin-induced lung fibrosis in mice. Int J Mol Sci 2018; 19: 2758.

34 Bleijerveld OB, Brouwers JFHM, Vaandrager AB, Helms JB, Houweling M. The CDP-ethanolamine pathway and phosphatidylserine decarboxylation generate different phosphatidylethanolamine molecular species. $J$ Biol Chem 2007; 282: 28362-28372.

35 Braverman NE, Moser AB. Functions of plasmalogen lipids in health and disease. Biochim Biophys Acta 2012; 1822: 1442-1452.

36 Honsho M, Fujiki Y. Plasmalogen homeostasis - regulation of plasmalogen biosynthesis and its physiological consequence in mammals. FEBS Lett 2017; 591: 2720-2729.

37 Malavolta M, Bocci F, Boselli E, Frega NG. Normal phase liquid chromatography-electrospray ionization tandem mass spectrometry analysis of phospholipid molecular species in blood mononuclear cells: application to cystic fibrosis. J Chromatogr B Analyt Technol Biomed Life Sci 2004; 810: 173-186.

38 van der Veen JN, Kennelly JP, Wan S, Vance JE, Vance DE, Jacobs RL. The critical role of phosphatidylcholine and phosphatidylethanolamine metabolism in health and disease. Biochim Biophys Acta Biomembr 2017; 1859 (9 Pt B): $1558-1572$.

39 Islam MN, Das SR, Emin MT, et al. Mitochondrial transfer from bonemarrow-derived stromal cells to pulmonary alveoli protects against acute lung injury. Nat Med 2012; 18: 759-765.

40 Babajani A, Hosseini-Monfared P, Abbaspour S, Jamshidi E, Niknejad H. Targeted mitochondrial therapy with over-expressed MAVS protein from mesenchymal stem cells: a new therapeutic approach for COVID-19. Front Cell Dev Biol 2021; 9: 695362.

41 Patananan AN, Wu TH, Chiou PY, Teitell MA. Modifying the mitochondrial genome. Cell Metab 2016; 23: 785-796.

42 Kheirollahi V, Wasnick RM, Biasin V, et al. Metformin induces lipogenic differentiation in myofibroblasts to reverse lung fibrosis. Nat Commun 2019; 10: 2987. License (http://creativecommons.org/licenses/by-nc-nd/4.0/). 